\title{
Antropometría como predictor de diabetes gestacional: Estudio de cohorte
}

\author{
ANDREA HUIDOBRO M. ${ }^{1}$, ANDREW M. PRENTICE ${ }^{2, \mathrm{a}}$, \\ ANTHONY, JC FULFORD ${ }^{2, \mathrm{~b}}$, JAIME ROZOWSKI N.,a
}

\section{Anthropometry as predictor of gestational diabetes mellitus}

Background: Gestational diabetes mellitus (GDM) is a high incidence disease. Easily measured predictor factors could help to implement preventive policies and early detection tests. Aim: To measure recognizable risk factors for GDM such as skinfolds and analyze the association between these factors and the development of GDM in a cohort of pregnant women. Material and Methods: Evaluation of 76 mothers that developed gestational diabetes, aged $32.2 \pm 0.6$ years and 324 control mothers that did not develop the disease, aged $30.1 \pm 0.3$ years. Weight, height, arm circumference, tricipital, bicipital, subscapular, suprailiac, knee, costal and mid-thigh skinfolds were measured in the pre-diseased stage. History of diabetes, fasting glucose and insulin levels were also evaluated. Results: Age, body mass index (BMI), fasting blood glucose, the homeostasis model assessment of insulin resistance (HOMA), bicipital, tricipital, costal, subscapular, suprailiac, and knee skinfolds were associated with GDM development. Age, fasting blood glucose and subscapular skinfolds were independent predictors in the logistic regression model. The odds ratio for a subscapular skinfold over percentile 90 was 1.7 (95\% confidence intervals: 1.07-3.04). Conclusions: Age and fasting blood glucose are independent risk factors for GDM. Subscapular skinfold is also an independent risk factor and could be used to detect high risk pregnant women and implement preventive policies.

(Rev Med Chile 2010; 138: 1373-1377).

Key words: Anthropometry; Diabetes, gestational; Skinfold thickness.

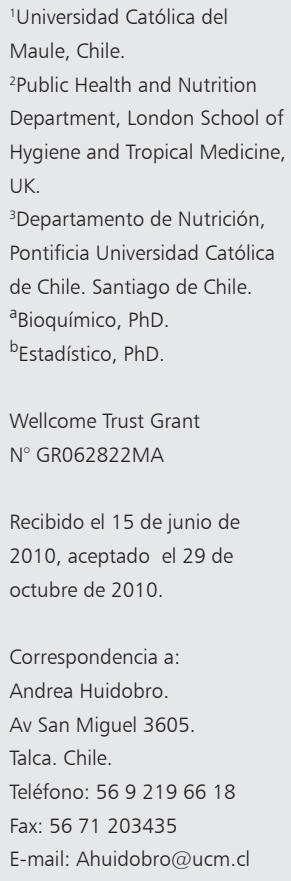

L a diabetes gestacional (DG) es una patología que ha ido aumentando su incidencia de la misma manera que la DM tipo 2, tanto en Chile como en el resto del mundo. La DG significa un riesgo para madre e hijo, a corto y largo plazo $^{1,2}$ y tanto su prevención como su diagnóstico y tratamiento precoces son esenciales para la mejorar el pronóstico del binomio madre-hijo ${ }^{3}$. El conocimiento de factores predictores modificables puede, por un lado, detectar una población en riesgo sobre la cual podemos intensificar las medidas preventivas, o bien, detectar una subpoblación en la que se justifica un enfrentamiento diagnóstico de un sólo paso, es decir, directamente realizar un test de tolerancia con 75 g de glucosa oral.
La detección precoz y el beneficio del tratamiento en DG justifica la necesidad de encontrar factores predictores de fácil medición. Estudios como el de Langer, comparando mujeres diabéticas diagnosticadas en la semana $37 \mathrm{y}$, por lo tanto, no tratadas, con mujeres diabéticas tratadas y con mujeres sin diabetes, refieren un beneficio del tratamiento expresado en 59\% de complicaciones en las diabéticas no tratadas, $18 \%$ de las tratadas y sólo $11 \%$ de complicaciones en las no diabéticas ${ }^{4}$. Otros estudios también han demostrado beneficios significativos del tratamiento intensivo de la DG, aunque los efectos han sido menores. En este sentido, es posible que el beneficio del tratamiento precoz sea más evidente al mediano y largo plazo, al disminuir 
las complicaciones crónicas asociadas con la resistencia insulínica, asociación poco estudiada en el largo plazo en mujeres que hayan presentado DG.

En cuanto a factores de riesgo antropométricos, sólo se han descrito al IMC y la relación cintura cadera como predictores de $\mathrm{DG}^{5-7}$. Sin embargo, el índice de masa corporal (IMC) es un indicador que pierde valor en el embarazo al verse alterado por edema y por el mismo componente materno-fetal del embarazo. Por otro lado, lo mismo ocurre con la razón cintura cadera, que no sería tan buen estimador de adiposidad central durante el embarazo. En este sentido, los pliegues cutáneos debieran ser mejores estimadores de adiposidad y, por lo mismo, mejores predictores de DG, cuyo componente mayor es la resistencia insulínica, la cual está directamente relacionada a obesidad abdominal.

En la búsqueda de fórmulas para estimar el componente graso corporal durante el embarazo, se ha determinado que el crecimiento de los pliegues cutáneos en el embarazo es diferencial. Se sabe, por ejemplo, que el incremento es mayor en el pliegue suprailíaco, seguido por el pliegue subescapular y costal. El incremento en el pliegue de medio muslo es también importante, no así el pliegue de rodilla y el tricipital ${ }^{8}$. En base a estos conocimientos y, a partir de la fórmula de Pipe ${ }^{9}$, que estimó la masa libre de grasa a partir del peso y del potasio total en la embarazada, un estudio en mujeres suecas, derivó ecuaciones para estimar la masa grasa en distintos estados del embarazo, con un coeficiente de correlación de 0,69 entre las semanas 16 y $18^{8}$.

Es en este contexto en el que se plantea estudiar los pliegues cutáneos medidos en etapa temprana del embarazo, y la estimación de grasa corporal derivada de estas mediciones, como predictores de DG en una cohorte de mujeres embarazadas.

\section{Metodología}

Se analizaron datos de 76 mujeres con diabetes gestacional y 324 controles de una cohorte de mujeres descrita en artículo previo ${ }^{10}$. La cohorte original estaba constituida por 77 mujeres que desarrollaron DG y 327 controles, sin embargo, para el estudio actual, se excluyeron aquellas que no poseían la totalidad de las mediciones antropométricas. La cohorte original consistió en mujeres cursando embarazo de hijo único de menos de 20 semanas al inicio del estudio y se siguieron con test de tolerancia oral a la glucosa en el segundo y tercer trimestre de embarazo para diagnosticar o descartar DG. Se evaluaron edad, antecedentes personales de diabetes, nivel de educación, número de embarazos previos, antecedentes familiares de diabetes, obesidad e hipertensión arterial, al ingreso al estudio. Se midió además talla y peso para cálculo de índice de masa corporal (IMC). Debido a que las mujeres ingresaron entre la semana 4 y la 20 de embarazo al estudio, esta variable podría actuar como confusor entre la asociación de DG e IMC. Por esto, se clasificó a las pacientes en normopeso o sobrepeso según su IMC y edad gestacional en base a la curva de Rosso Mardones ${ }^{11}$. El análisis antropométrico incluyó la medición de circunferencia de brazo, pliegues tricipital, bicipital, subescapular, suprailíaco, rodilla, costal y medio muslo. A partir de la ecuación de Forsum $^{8}$, se estimó la grasa corporal de las pacientes. Para las mediciones antropométricas se utilizó una cinta métrica flexible no distensible. La circunferencia de brazo se midió con la cinta métrica horizontal en el punto medio entre el acromion y el olécranon con la paciente de pies y relajada. Los pliegues fueron medidos con un cáliper Holtain a un centímetro y en ángulo recto en el hemicuerpo dominante, cada uno fue medido tres veces y se tomó como valor el promedio de estas tres mediciones. El pliegue suprailíaco se tomó inmediatamente sobre la cresta ilíaca hacia abajo y adelante en la línea media axilar. El pliegue subescapular se midió un $\mathrm{cm}$. bajo el ángulo inferior de la escápula con pliegue dirigido hacia abajo y lateral en $45^{\circ}$. El pliegue costal se midió en la intersección de la línea horizontal trazada en el borde distal del esternón con la línea vertical axilar media. El pliegue tricipital se midió verticalmente en el punto medio entre el acromion y el olecranon en la parte posterior del brazo. El pliegue bicipital se midió en el mismo nivel del tricipital, pero en la cara anterior. El pliegue de medio muslo se midió verticalmente en el punto medio entre la rótula y el pliegue inguinal con el peso del cuerpo cargado al lado opuesto. El pliegue de rodilla se midió sobre la rótula, con el caliper en posición vertical. Se evaluó además resistencia insulínica mediante Homeostasis model assessment $(\mathrm{HOMA})^{12}$, para lo cual se midieron glicemia e insulinemia basal. La glicemia se midió por el método de glucosa oxidasa y la insulinemia, con radioinmunoensayo (Coat-A 
Antropometría y diabetes gestacional - A. Huidobro M. et al

insulin kit, Diagnostic Products Corporation, Los Angeles, U.S.A).

El análisis estadístico presenta las variables en porcentaje con intervalo de $95 \%$ de confianza o media con desviación estándar. La asociación entre DG y los factores de riesgo y pliegues se evaluaron en análisis univariado con test de t de Student. La fuerza de asociación de las variables se evaluó con OR y su respectivo intervalo de $95 \%$ de confianza en regresión logística simple. Para el análisis de OR se expresó la asociación en base a desviaciones estándar de cada variable, para permitir comparar efectos. La independencia de los factores de riesgo significativamente asociados a DG se evaluó con regresión logística paso a paso. La fuerza de asociación de aquellos pliegues cutáneos que resultaran significativamente asociados al desarrollo de DG se analizó además según percentiles, con el objetivo de proponer un punto de corte que represente un riesgo aumentado de desarrollar DG. Se calculó además sensibilidad, especificidad y valores predictivos de los pliegues que demostraran independencia, con el corte en el percentil 90. El análisis estadístico se realizó utilizando el programa estadístico STATA 9.0.

La aprobación ética del estudio se obtuvo de la London School of Hygiene and Tropical Medicine y de la Pontificia Universidad Católica de Chile. Todas las mujeres firmaron consentimiento informado antes de ser reclutadas.

\section{Resultados}

Se analizaron 76 mujeres con DG y 324 controles. La incidencia de DG en esta cohorte fue de 19\%. Las características de ingreso de las mujeres al estudio, con DG y controles, se presentan en la
Tabla 1. La edad de las mujeres que desarrollaron DG fue de 32,2 $\pm 0,6$ años y la de los controles fue de 30,05 $\pm 0,27$ años $(p=0,008)$. El IMC al ingreso de las mujeres con DG fue de $28,9 \pm 0,5 \mathrm{~kg} / \mathrm{m}^{2}$ y el de los controles, de $26,5 \pm 0,3 \mathrm{~kg} / \mathrm{m}^{2}(\mathrm{p}=0,003)$. Según estado nutricional de acuerdo a las tablas de Rosso y Mardones ${ }^{11}$, 24,3\% de las mujeres con sobrepeso u obesidad desarrollaron DG, mientras que $14 \%$ de las normopeso desarrolló DG ( $\mathrm{p}=$ 0,008). Las mujeres con DG mostraron menor grado de educación $(\mathrm{p}=0,036)$ y mayor número de embarazos previos $(p=0,004)$. Sin embargo, el número de embarazos previos no mostró diferencia significativa después de ajustar por edad ( $\mathrm{p}$ $=0,15)$. Entre los antecedentes familiares, sólo la hipertensión arterial se asoció al desarrollo de DG, desarrollándose en $23 \%$ de mujeres con antecedentes de hipertensión familiar y en 13,8\% de las mujeres sin este antecedente familiar $(\mathrm{p}=0,046)$.

En cuanto a valores antropométricos, la composición corporal estimada por la formula de Forsum, mostró un promedio de masa grasa de $34,4 \pm 4,3 \%$ (IC 95\%: 24,3 a 48,1\%), en el primer trimestre del embarazo. La correlación entre porcentaje de grasa según fórmula de Forsum e IMC fue $\mathrm{R}=0,8$. El porcentaje de grasa estimado por fórmula se asoció a desarrollo de DG $(35,8 \%$, CI 34,9 a 36,7\% en DG vs $34,4 \%$, CI 33,9 a $34,9 \%$ en controles).

En cuanto al análisis de los pliegues individuales, se encontró una asociación significativa entre DG y pliegues tricipital, subescapular, costal y suprailíaco, según se muestra en Tabla 2.

Con relación a valores de glicemia, insulinemia y cálculo de HOMA, sólo glicemia y HOMA se relacionaron positivamente al desarrollo de DG con OR de 1,55 (95\% IC: $1,18-2,03)$ y $2,19(95 \%$ IC 1,02-4,69) respectivamente.

Tabla 1. Características al ingreso, con menos de 20 semanas de embarazo, de mujeres que desarrollaron DG y controles

\begin{tabular}{|lrrrrc|}
\hline & DG & IC 95\% & Controles & IC 95\% & Valor p \\
\hline Edad $(x \pm d s)$ & $32,3 \pm 0,60$ & $31,1-33,5$ & $30,4 \pm 0,27$ & $29,9-31,0$ & 0,0037 \\
\hline IMC $(x \pm d s)$ & $26,1 \pm 0,60$ & $24,9-27,3$ & $25,1 \pm 0,24$ & $24,6-25,5$ & 0,0650 \\
\hline Edad gestacional al ingreso & $15,53 \pm 0,46$ & $14,6-16,4$ & $15,58 \pm 0,20$ & $15,2-16,0$ & 0,9033 \\
Glicemia basal & $81,36 \pm 1,20$ & $79,0-83,8$ & $76,54 \pm 0,49$ & $75,6-77,5$ & 0,0000 \\
\hline HOMA $(x \pm d s)$ & $2,80 \pm 0,82$ & $1,16-4,43$ & $1,72 \pm 0,05$ & $1,63-1,81$ & 0,0009 \\
Insulina basal & $12,33 \pm 2,60$ & $7,15-17,51$ & $9,02 \pm 0,20$ & $8,62-9,42$ & 0,0133 \\
\hline
\end{tabular}


Tabla 2. Relación entre pliegues cutáneos y desarrollo de DG en mujeres embarazadas chilenas con regresión linear simple

\begin{tabular}{|c|c|c|c|c|c|c|}
\hline & $\begin{array}{l}\text { Controles } \\
(n=324)\end{array}$ & $\begin{array}{c}\text { DG } \\
(n=76)\end{array}$ & $\begin{array}{l}\text { Regresión } \\
\text { logística OR }\end{array}$ & Valor P & $95 \%$ & o IC \\
\hline Pliegue bicipital & $13,2 \pm 0,28$ & $14,3 \pm 0,56$ & 1,25 & 0,066 & 0,99 & 1,58 \\
\hline Pliegue tricipital & $22,7 \pm 0,33$ & $25,0 \pm 0,69$ & 1,51 & 0,001 & 1,17 & 1,95 \\
\hline Pliegue subescapular & $24,9 \pm 0,42$ & $28,4 \pm 0,80$ & 1,56 & 0,001 & 1,20 & 2,03 \\
\hline Pliegue costal & $21,6 \pm 0,40$ & $24,4 \pm 0,68$ & 1,44 & 0,005 & 1,12 & 1,86 \\
\hline Pliegue suprailíaco & $24,9 \pm 0,43$ & $28,2 \pm 0,81$ & 1,61 & 0,000 & 1,22 & 2,11 \\
\hline Pliegue medio muslo & $28,4 \pm 0,38$ & $30,0 \pm 0,79$ & 1,29 & 0,053 & 1,00 & 1,66 \\
\hline Pliegue rodilla & $13,3 \pm 0,29$ & $13,8 \pm 0,72$ & 1,08 & 0,553 & 0,85 & 1,37 \\
\hline
\end{tabular}

Expresado como desviaciones estándar.

Tabla 3. Modelo de regresión para edad, variables antropométricas y glicemia basal y desarrollo de DG

\begin{tabular}{|lllll|}
\hline DG & OR & $\mathbf{P}>|\mathbf{z}|$ & \multicolumn{2}{c|}{ IC $\mathbf{9 5 \%}$} \\
\hline Edad & 1,33 & 0,039 & 1,01 & 1,75 \\
Pliegue subescapular & 1,5 & 0,003 & 1,15 & 1,99 \\
Glicemia basal & 1,4 & 0,008 & 1,10 & 1,86 \\
Const & 0,21 & 0,000 & 0,16 & 0,27 \\
\hline
\end{tabular}

Expresado en unidades de desviación estándar.

En el análisis de regresión logística paso a paso, sólo las variables edad, pliegue subescapular y glicemia basal demostraron ser independientes en su asociación con DG (Tabla 3).

El percentil 90 del pliegue subescapular fue $35 \mathrm{~mm}$. El OR de esta variable considerada como dicotómica en el percentil 90, fue de 1,7 con intervalo de 95\% de confianza entre 1,07 y 3,04 (p $=0,029)$. La sensibilidad del pliegue subescapular sobre percentil 90 fue de $62 \%$ y la especificidad de $74,5 \%$, con una razón de verosimilitud positiva de 2,43 y razón de verosimilitud negativa de 0,51 . La sensibilidad para IMC de 25 o más fue de 56,6\%, especificidad de $55,2 \%$ y valor predictivo positivo de $22,9 \%$.

\section{Discusión}

Este estudio es pionero en medir antropometría como predictor de DG y el primero en estimar asociación de pliegues individuales e integrados en fórmula de Forsum de estimación de grasa corporal. Los resultados apoyan a la edad, el sobrepeso y la glicemia basal como factor de riesgo de DG, asociación descrita en estudios nacionales y extranjeros ${ }^{6,13}$. Edad y glicemia basal fueron factores de riesgo independientes. Si bien el sobrepeso medido según IMC es un factor de riesgo, no resultó un factor independiente en este estudio. Por otro lado, los pliegues cutáneos, en particular el pliegue subescapular, fue un factor predictor independiente de la edad y de la glicemia basal. Este estudio no es el primero en determinar que otra medición antropométrica es mejor predictor de DG que el IMC, aunque sí el primero en describir pliegues cutáneos como predictores. Un estudio en 106 mujeres embarazadas demostró que la razón cintura cadera es mejor predictor de DG que el $\mathrm{IMC}^{7}$ con un OR de 12,5 (IC 95\%: $1,82-77,43)$, aunque las mujeres con mayor índice de cintura cadera también subieron más de peso, lo que podría estar actuando como confundente.

El pliegue subescapular fue el mejor predictor de DG. Esta asociación es explicable, dado que el pliegue subescapular es un buen estimador de adiposidad central, que es la más asociada a insulino resistencia, factor decisivo en la generación de diabetes gestacional. Por otro lado, el IMC durante el embarazo puede estar influenciado por edema y no es tan buen estimador de de grasa corporal como los pliegues cutáneos. Si bien, los pliegues cutáneos son buenos estimadores de grasa corporal, su medición requiere tiempo y dependen del operador, necesitando cierto grado de entrenamiento. Por esta razón es muy promisorio que 
Antropometría y diabetes gestacional - A. Huidobro M. et al

un solo pliegue, de relativa fácil medición como el subescapular, sea capaz de predecir DG. Aunque las curvas de Rosso y Mardones se crearon para medir sobrepeso en embarazadas chilenas, la asociación entre sobrepeso medido según estas tablas y el desarrollo de DG no fue independiente, por lo que no sería mejor predictor de DG y su uso no estaría apoyado por este estudio.

\section{Conclusión}

El pliegue subescapular es predictor de DG y puede ser utilizado como rutina para detectar mujeres en riesgo cursando embarazo de hasta 20 semanas.

Agradecimientos: Este estudio fue financiado por el Wellcome Trust, entidad que financió exclusivamente el trabajo en terreno. Agradecemos al equipo de nutricionistas y enfermeras que apoyaron en la Pontificia Universidad Católica de Chile durante el desarrollo de este proyecto.

\section{Referencias}

1. Jovanovic L, Knopp R, Kim H, Cefalu W, Zhu X, Lee Y, et al. Elevated pregnancy losses at high and low extremes of maternal glucose in early normal and diabetic pregnancy: evidence for a protective adaptation in diabetes. Diabetes Care 2005; 5: 1113-7.

2. Jensen DM, Sorensen B, Feilberg-Jorgensen N, Westergaard JG, Beck-Nielsen H. Maternal and perinatal outcomes in 143 Danish women with gestational diabetes mellitus and 143 controls with a similar risk profile. Diabet Med 2000; 4: 281-6.

3. Yogev Y, Metzger B, Hod M. Establishing the diagnosis of gestational diabetes mellitus: Impact of the hypergly- cemia and adverse pregnancy outcome study. Seminars in Fetal \& Neonatal Medicine 2009; 2: 94-100.

4. Langer O, Yogev Y, Most O, Xenakis E. Gestational diabetes: the consequences of not treating. Am J Obstet Gynecol 2005; 4: 989-97.

5. Torloni MR, Beltrán AP, Horta AR, Nakamura MU, Attalah AN, Moron AF, et al. Prepregnancy BMI and the risk of gestational diabetes: a systematic review of the literature with meta-analysis. Obesty reviews 2009; 2: 194-203.

6. Yeung EH, Hu F, Solomon CG, Chen L, Louis GM, Schisterman E, et al. Life-course weight characteristics and the risk of gestational diabetes. Diabetología 2010; 4: 668-78.

7. Madhavan A. A pilot study on the usefulness of body mass index and waist hip ratio as a predictive tool for gestational diabetes in Asian Indians. Gynecological Endocrinology 2008; 12: 701-7.

8. Forsum E, Sadurskis A, Wager J. Estimation of body fat in healthy Swedish women during pregnancy and lactation. Am J Clin Nutr 1989; 3: 465-73.

9. Pipe N, Smith T, Halliday D, Edmonds C, Williams C, Coltart T. Changes in fat, fat-free mass and body water in human normal pregnancy. Br J Obstet Gynaecol 1979; 12: 929-40.

10. Huidobro A, Prentice A, Fulford A, Parodi C, Rozowski J. [Gestational diabetes, comparison of women diagnosed in second and third trimester of pregnancy with non GDM women: Analysis of a cohort study]. Rev Med Chile 1010; 3: 316-21.

11. Mardones F, Rosso P. [Design of a weight gain chart for pregnant women]. Rev Med Chile 1997; 12: 1437-48.

12. Cohen O, Epstein G, Weisz B, Homko C, Sivan E. Longitudinal assessment of insulin sensitivity in pregnancy. Validation of the homeostasis model assessment. Clin Endocrinol (Oxf) 2006 6: 640-4.

13. Huidobro A, Fulford A, Carrasco E. Prevalencia de diabetes gestacional y su relación con obesidad en embarazadas Chilenas. Rev Med Chile 2004; 8: 931-8. 